\title{
REPRESENTING A DIFFERENTIABLE FUNCTION AS A CARTESIAN PRODUCT
}

\author{
MICHAEL R. COLVIN
}

\begin{abstract}
This article produces an elementary proof of a result originally stated without proof by J. Leray. The main result gives conditions so that a continuously differentiable map from a product neighborhood of the origin in $\mathbf{R}^{n}$ into $\mathbf{R}^{n}$ can be homotoped to a cartesian product of maps on intervals. The resulting product function preserves properties of the original map near the origin.
\end{abstract}

Introduction. The purpose of this note is to provide an argument of a theorem orginally stated without a proof by J. Leray in [Le, p. 215]. As a step in his development of the uniqueness of the fixed point index, R. F. Brown uses the statement of Leray's theorem [ $\mathbf{B}_{\mathbf{1}}$, Lemma 9, p. 555; $\mathbf{B}_{\mathbf{2}}$, Lemma 7, p. 168; and $\mathbf{B}_{\mathbf{3}}$, Lemma 7.6, p. 15]. During a review of Brown's construction the author observed that the argument, which is the same in all cases, is incorrect. Leray simply records the statement without proof and Brown assumes the result is a well-known fact. We write this note in the spirit set by Brown in $\left[\mathbf{B}_{1}\right]$, that is, the argument is "elementary", needing only information from elementary differential topology, linear algebra and the calculus. The author wishes to extend his appreciation to the fixed point group at California State University, San Luis Obispo, California, for comments on an earlier version of this note. The article was written while the author was visiting the University of Alabama, University, Alabama.

1. Some lemmas. In this section, we collect some lemmas which will be used to establish our main result. We need a result which follows from the Whitney extension theorem [AR, W]. Let $A$ be a finite polyhedron contained in $\mathbf{R}^{n}$. Assume $h_{0}: A \rightarrow R^{n}$ is a continuous map. We shall refer to $h_{0}$ as being class $C^{1-}$ provided there exists a continuous map $h_{1}: A \rightarrow L\left(\mathbf{R}^{n}, \mathbf{R}^{n}\right)$ so that the maps

$$
R_{0}(x, y)=h_{0}(x)-h_{0}(y)-h_{1}(x)(x-y)
$$

and

$$
R_{1}(x, y)=h_{1}(y)-h_{1}(x)
$$

satisfy: for each $x_{0} \in A$ and $k=0$ or $k=1$

$$
\left|R_{k}(x, y)\right| /\|x-y\|^{1-k} \rightarrow 0 \quad \text { as } x, y \rightarrow x_{0} .
$$

Received by the editors December 22, 1982 and, in revised form, February 28, 1983.

1980 Mathematics Subject Classification. Primary 55M20; Secondary 15A04.

Key words and phrases. Fixed point theory, index, jacobian, Whitney extension theorems, inverse function theorem. 
1.1. Lemma [W, Lemma 2, p. 69]. Assume $h_{0}: A \rightarrow R^{n}$ is of class $C^{1-}$ on the finite polyhedron $A$. Then there is a continuously differentiable function $H: \mathbf{R}^{n} \rightarrow \mathbf{R}^{n}$ such that $D^{k} H_{1 A}=h_{k}$ for $k=0$ or 1 .

We shall use the standard notation $L(n)$ and $G L(n)$ to denote the collection of linear transformations and invertible linear transformations, respectively. We also recall that topologically $G L(n)$ is an open subset of $L(n)$ and consists of two path components $G L^{+}(n)$ and $G L^{-}(n)$ determined by the sign of the determinant.

We let $S(n)$ denote the symmetric group on $n$ symbols. If $A=\left(a_{i j}\right)$ is an $n \times n$ matrix, we recall that the determinant of $A$ can be computed by

$$
\operatorname{det} A=\sum_{\phi \in S(n)} \sigma(\phi) a_{1, \phi(1)} \cdots a_{n, \phi(n)},
$$

where $\sigma(\phi)$ is the sign of the permutation $\phi$. If $\operatorname{det} A \neq 0$, then an observation of the above expression yields a permutation $\phi \in S(n)$ so that $\sigma(\phi) a_{1, \phi(1)} \cdots a_{n, \phi(n)} \neq 0$, and, if $B_{\phi}$ is the matrix $\left(b_{i, j}\right)$ with

$$
b_{i, j}= \begin{cases}0 & \text { if } j \neq \phi(i), \\ a_{i, \phi(i)} & \text { otherwise, }\end{cases}
$$

then $\phi$ can be chosen so that $B_{\phi}$ and $A$ lie in the same path component of $G L(n)$. We can therefore state:

1.2. LeMMA. If $A=\left(a_{i j}\right)$ is a nonsingular matrix, then there exist $\phi \in S(n)$ and $a$ continuous map $H: I \rightarrow G L(n), I=[0,1]$, so that $H(0)=A$ and $H(1)=B_{\phi}=\left(b_{i, j}\right)$, with $b_{i, j}$ given by (1.10).

2. Main theorem. Given a space $X$ and a function $f: X \rightarrow \mathbf{R}^{n}$, we will always denote by the corresponding capital letter $F: X \rightarrow \mathbf{R}^{n}$ the function $F(x)=x-f(x)$ for all $x \in X$. If $U \subseteq \mathbf{R}^{n}$ is an open subset of $\mathbf{R}^{n}$ and $f: U \rightarrow \mathbf{R}^{n}$ is a differentiable map, we will use the notation $J f(x)$ to denote the jacobian of $f$ at $x$, i.e.,

$$
J f(x)=\operatorname{det}\left(\partial f_{j} / \partial x_{j}\right), \quad 1 \leqslant i \leqslant n, 1 \leqslant j \leqslant n .
$$

2.1. Main Theorem. Suppose $0 \in\left(a_{j}, b_{j}\right) \subseteq \mathbf{R}, j=1, \ldots, n$, let $X=\prod_{j=1}^{n}\left[a_{j}, b_{j}\right]$. If $f: X \rightarrow X$ is a map such that $f(0)=0, f$ is $C^{1}$ on some neighborhood of 0 and $J F(0) \neq 0$, then there exists a homotopy $h: I \rightarrow \operatorname{Map}(X)$ and an open subset $V$ of $X$, $0 \in V$, such that:

(a)

$$
h(t)(x)= \begin{cases}f(x), & t=0 \\ \left(\prod_{j=1}^{n} g_{j}\right)(x), & t=1\end{cases}
$$

where $g_{j}:\left[a_{j}, b_{j}\right] \rightarrow\left[a_{j}, b_{j}\right]$ are continuous maps;

(b) $h(t)(0)=0$ for all $t \in I$;

(c) if $J: I \rightarrow \mathbf{R}$ is defined by $J(t)=J(H(t))(0)$, then $J$ is a continuous nonvanishing function of $t$;

(d) $H(t)$ is one-to-one on $V$ for all $t \in I$. 
Proof. Let $F_{i j}=-\partial f_{i} / \partial x_{j}, i \neq j$ and $F_{i i}=1-\partial f_{i} / \partial x_{i}$, each partial taken at $0 \in \mathbf{R}^{n}$. Using the assumption $J F(0) \neq 0$ and applying Lemma 2 , there exists a permutation $\phi \in S(n)$ so that $\sigma(\phi) F_{1, \phi(1)} \cdots F_{n, \phi(n)} \neq 0$ and, for $b_{i, j}=F_{i, \phi(i)}, \phi(i)$ $=j$ and $b_{i, j}=0$ otherwise, the matrices $B_{\phi}=\left(b_{i, j}\right)$ and $\left(F_{i, j}\right)$ are in the same path component of $G L(n)$.

For $1 \leqslant j \leqslant n$ and $\phi \in S(n)$ chosen as above, define $g_{j}:\left[a_{j}, b_{j}\right] \rightarrow\left[a_{j}, b_{j}\right]$ by $g_{j}\left(x_{j}\right)=f_{k}\left(0, \ldots, x_{j}, \ldots, 0\right)$ with $\phi(j)=k$. We denote by $g$ the function, $\prod_{j=1}^{n} g_{j}$, i.e., $g\left(x_{1}, \ldots, x_{n}\right)=\left(g_{1}\left(x_{1}\right), \ldots, g_{n}\left(x_{n}\right)\right)$. Using $G(x)=x-g(x)$, we observe $G$ is $C^{1}$ on some neighborhood of 0 and the jacobian matrix $\left(G_{i, j}\right)$ for $G$ at 0 is given by $B_{\phi}$. We can therefore construct a map $H_{0}: I \rightarrow G L(n)$ so that $H_{0}(0)=\left(F_{i j}\right)$ and $H_{0}(1)=\left(G_{i j}\right)$.

Let $W_{0} \subseteq X, 0 \in W_{0}$, be some neighborhood of 0 over which $f$ is differentiable. Pick $W \subseteq W_{0}$, so that $0 \in W, W$ is open $n$-cell and $\bar{W} \subseteq W_{0}$. We denote by $A$ the subset of $\mathbf{R}^{n+1}$ defined by $A=\bar{W} \times\{0\} \cup \bar{W} \times\{1\} \cup\{0\} \times I$. Given $(x, t) \in A$ define the continuous functions $\bar{h}: A \rightarrow X$ and $\bar{H}_{1}: A \rightarrow L(n)$ by

$$
\bar{h}(x, t)= \begin{cases}f(x), & t=0 \\ g(x), & t=1, \\ 0, & x=0 \text { and } 0 \leqslant t \leqslant 1,\end{cases}
$$

and

$$
\bar{H}_{1}(x, t)= \begin{cases}\left(F_{i j}\right)_{\uparrow, x}, & t=0 \\ \left(G_{i j}\right)_{\uparrow, x}, & t=1 \\ \left(H_{0}(t)\right)(0) & \text { otherwise. }\end{cases}
$$

We recall the notation $\bar{H}(x, t)=I(x)-\bar{h}(x, t)$. Using Lemma 1.1 on $H$ and $H_{1}$, we can construct a $C^{1}$ extension of $\bar{H}$, say $H$, mapping $\bar{W} \times I$ into $X$ so that $H(x, t)=\bar{H}(x, t)$ and $\left(H_{i, j}\right)_{\uparrow(x, t)}=\bar{H}_{1}(x, t)$ for all $(x, t) \in A$. Recall we have assumed $\bar{W}$ is an $n$-cell, hence there exists a radial retraction $\rho: X \rightarrow \bar{W}$. By defining $H=H \circ(\rho \times$ id $)$ we can extend $H$ to $X \times I$ and thereby construct a map $h$ : $i \rightarrow \operatorname{map}(X)$ by $H(x, t)=I(x)-h(t)(x)$.

By construction $h$ satisfies (a) and (b). For (c) we observe for $t \in I$ that $J(t)=J(H(t))(0)=J\left(\bar{H}_{1}(t)\right)(0)=J\left(H_{0}(t)\right)(0)$, therefore $J(t)$ is continuous and nonvanishing by the definition of $H_{0}$.

It remains to find a single neighborhood $V$ of 0 on which each $H(t)$ is one-to-one. Let $t \in I$. Then $J(t) \neq 0$ implies there exists an open subset, $V_{t} \subseteq W$, containing 0 and an open interval $I_{t} \subseteq I$ containing $t$, so that $D H(x, t)$ is nonsingular in $V_{t} \times I_{t}$. Using the compactness of $I$ there exists $\left\{t_{1}, \ldots, t_{p}\right\} \subseteq I$ so that $I \subseteq \cup_{i=1}^{p} I_{t_{i}}$. Define $V=\bigcap_{i=1}^{p} V_{t_{i}}$ and observe, if $(x, t) \in V \times I$, that $D H(x, t)$ is nonsingular and the Inverse Function Theorem implies $H(t)$ is one-to-one on $V$.

\section{REFERENCES}

[AR] R. Abraham and J. Robbin, Transversal mappings and flows, Benjamin, New York, 1967.

[B] R. F. Brown, An elementary proof of the uniqueness of the fixed point index, nacific J. Math. 35 (1970), 549-558. 


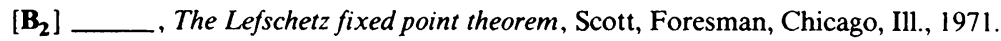

$\left[\mathbf{B}_{3}\right] \ldots$ Notes on Leray's index theory, Adv. in Math. 7 (1971), 1-28.

[Le] S. Leray, Sur les équations et les transformations, J. Math. Pures Appl. 24 (1945), 201-248.

[W] H. Whitney, Analytic extensions of differentiable functions defined on closed sets, Trans. Amer. Math. Soc. 36 (1934), 63-89.

Department of Mathematics, University of Alabama, University, Alabama 35486

Current address: Department of Mathematics, California State University, San Luis Obispo, California 93407 